\title{
Network testing strategies for local mobility service
}

\author{
R. Pignatta, D. Turco, P. Marchese \\ CSELT \\ Via Reiss Romoli 274, Torino, Italy, Tel. +39112285555 , \\ Fax +39 11228 5069, E-Mail Roberto.Pignatta@cselt.stet.it \\ F. Corno \\ Italtel \\ Castelletto di Settimo M.se (MI), Italy, Tel. +3924388 8708 \\ Fax+3924388 8245, E-Mail cornofab@ic8u82.settimo.italtel.it \\ G. Raneri \\ Telecom Italia \\ Via di Valcannuta 250, Roma, Italy, Tel. +39636885690 \\ Fax +39636885727
}

\begin{abstract}
Cordless Mobility may be regarded as a new PSTN service which allows customers to use their private cordless terminals in a public domain area, typically the urban circle. New functionalities are required to switching systems and to the network itself, as a wireless access, Intelligent Network nodes availability, mobile call management, including location register and terminal authentication capabilities. The paper presents the testing approach chosen to verify in a laboratory environment a prototypal CM solution based on DECT access to Italtel UT100 exchanges and an IN infrastructure, highlighting the necessity of a comprehensive testing strategy (protocol conformance, end to end service testing, system performance evaluation) and new testing tools for network integration activities.
\end{abstract}

DECT, interoperability, cordless mobılity.

\section{Keywords}

\section{INTRODUCTION}

In the international TLC community growing interest exists for Cordless Terminal Mobility (CTM) service. The service allows customers to use advanced cordless systems, such as DECT and CT2, not only in private/residential environments, but also in public or office domains, still preserving a personal profile. Several activities (e.g. within ETSI NA and RES3, Eurescom P507, DECT Operator's Group) and trials by most network Operators and switching manufacturers are in progress to investigate the bust way to use radio technologies and the means of connecting cordless terminals into PSTN through Intelligent Network capabilities (see [1] and [2]).

In this framework, a preliminary solution for CTM or Cordless Mobility (CM), service is proposed by Italtel (see [2] and [8]), the UT 100 switching system. It alian manufacturer. This 
solution is based on DECT access to local switches and improved IN capabilities. This paper focuses on describing the testing activities performed by CSELT, the corporate research centre of the STET holding, Italtel and Telecom Italia in order to verify, in a controlled environment (test plant), the correct operation of network nodes, wireless equipment and application programs.

\section{A PRELIMINARY SOLUTION FOR CM IMPLEMENTATION}

\subsection{Network Infrastructure}

The preliminary solution for a local mobility service proposed by Italtel (see [1], [2] and [8]) is based on the integration at Local Exchange (LE) level of a DECT access and the use of dedicated intelligent nodes (HDB, Home Data Base) as shown in the Figure 1. The solution relies upon the availability, in the current Italian Intelligent Network, of SSP (Service Switching Point) capabilities on all main access switches.

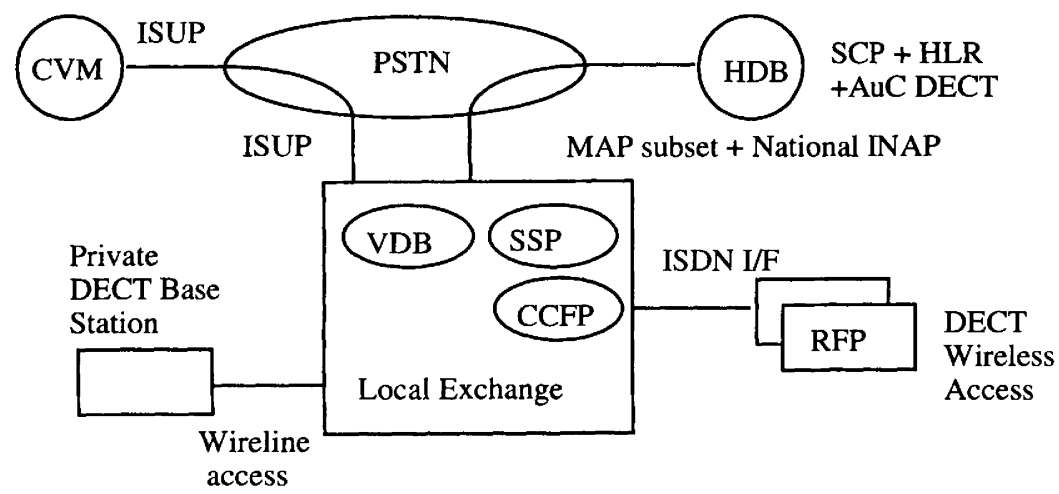

Figure 1 A reference network configuration for $\mathrm{CM}$ service.

DECT Radio Fixed Parts (RFPs) are directly connected to the switching system using standard copper pairs and a proprietary interface based on ISDN BRA. The exchange is upgraded with a number of new capabilities (referred to as CCFP, Common Control Fixed Part), including DECT (layers 2 and 3) protocol management, bit rate conversion from ADPCM $32 \mathrm{kbps}$ to standard $64 \mathrm{kbps}$, remote powering of RFP, Visitor Data Base (VDB) functionalities. In the lack of international standards, a combination of Mobile Application Part (MAP) [9] and the national IN Application Part (INAP) is used to dialogue, using standard CCS7 connections, with the HDB.

The HDB is needed to provide both terminal mobility data and customer profiles. As such, it incorporates Service Control Point (SCP) capabilities, Home Location Register (HLR) capabilities and Authentication Centre (AuC) functionalities. HLR and AuC solutions are derived from the current GSM implementation, but need adaptations to cope with DECT access requirements.

The proposed network configuration may also include a Centralized Voice Mail (CVM) system, which unsuccessful calls to roaming users are directed to. 


\subsection{Basic Service Features}

In the proposed solution, customers use a single portable Generic Access Profile (GAP) [4] DECT handset for both wireless (via public RFP) and wireline (via a private cordless base station) network access. As the customer switches on his handset inside the public radio coverage, or moves from one location area to another one, the handset automatically updates his position in the network by changing his location information in the HDB. When the user reaches the radio coverage of his private base station (normally placed in a residential or office environment), the terminal can be used as a standard cordless, with charging advantages.

The CM customer is associated to a single telephone number, regardless of the underlying access method (wireless or wireline) to the network. Number translation to the roaming number (used when the user is registered in the public domain), or to the wireline telephone number (used when the user is registered in the private home domain), is made by the HDB. In public environment, when the handset is off or out of radio coverage, incoming calls may be routed to a Centralized Voice Mail.

The proposed solution offers handover capabilities as well, to ensure voice connectivity when moving to new RFPs during conversation. In line with ETSI DECT GAP standards, both bearer and connection handover are fully supported.

Security aspects include standard DECT features as handset authentication (with a cryptographic challenge-response mechanism) and data confidentiality (by means of a key stream used for the encryption of data at DECT MAC layer).

The proposed service profile may comprise a number of additional services and features such as Centralized Voice Mail subscription and activation, call barring, call forwarding. They are offered using IN capabilities.

\section{Simple Call Flow}

A simple call flow is shown in the Table 1 below to demonstrate the signalling call processing for a mobile terminating call, involving no additional features, to a CM user registered at a public RFP.

In the following each item describe, in the right order, the signalling call procedures involving all network elements (RFP, HDB, Local Exchange, PT) for a mobile terminated call scenario.

1. The calling user dials the telephone number of the called user and the call is routed to the PSTN local exchange (SSP) via a IAM ISUP-S message.

2. The SSP recognizes the CM call and sends an INAP Provide Instruction query to the intelligent node $\mathrm{HDB}$.

3. The HDB sends a MAP Provide Roaming Number operation to the visited Local Exchange (the exchange which covers the geographic area where the user is roaming in) and gets a Roaming Number (an E164 number, dynamically assigned by the visited exchange to reach the CM user) which is transferred to the SSP via an INAP Connection Control Connect operation.

4. The SSP routes the call to the visited local exchange using the Roaming Number as the terminating address.

5. The visited local exchange pages the user. When the CM user's handset is located, the local exchange authenticates the handset, activates ciphering procedures, completes the call setup and provides audible ring-back to the caller.

Additional messages are required if, for instance, the CM user doesn't answer to the incoming call (which results in a call forwarded to the Centralized Voice Mail). 
Table 1 A simple Cordless Mobility call flow example

Call flow legend:

Link/Trunk signalling
.

.......... Radio signalling

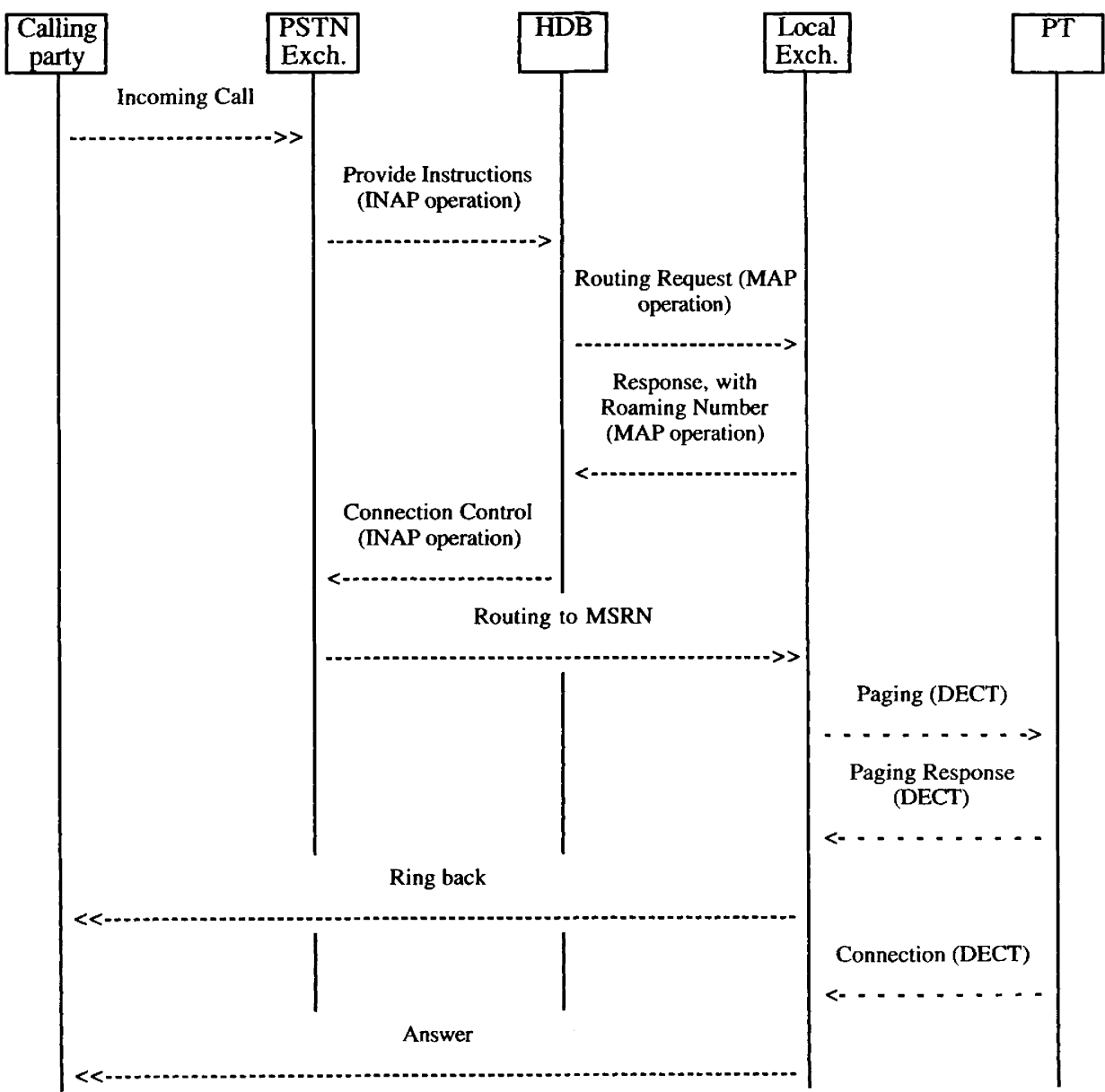

\section{CORDLESS MOBILITY TESTING CHALLENGES}

The verification, even in a prototypal environment, of a complex service as that described above, requires a comprehensive testing program to inspect each network element, in particular 
the RFP, the LE and the databases, ensure that systems would interoperate each other, and verify that the users could appropriately access the service using standard terminals. The approach to Cordless Mobility testing must cope with new challenges with respect to "traditional" switch based service testing needs, briefly summarized below.

1 Integration of wireless and wireline access to switching system.

The possibility to use the same terminal in both private (using a wireline network access) and public (using a radio network access) environment increases the complexity in service testing (mobility aspects, compatibility with subscribed service features, environment switch procedures).

2 Interaction between different protocols in the network.

Integration of Mobile Application Protocols (as GSM MAP) and IN Application Protocols (as the CS-1 like INAP protocol used in the Italy IN) in field applications needs appropriate test configurations. The proposed CM service, moreover, as briefly described above, includes interactions between INAP, MAP, ISUP and DECT protocols, with complex interactions which need comprehensive end to end verifications.

\section{$3 \quad$ Use of newly released portable terminal.}

The proposed Cordless Mobility service is based on the availability of DECT terminals with GAP profile. However, terminals are still prototypal and not completely certified as the relevant standards are not officially approved yet. This adds complexity in isolating problems in the network.

$4 \quad$ Introduction of new network systems (RFPs and DECT based HDB).

New network systems, as an HDB tailored to DECT authentication procedures, and equipments, as DECT RFPs, are necessary for the deployment of the service. In the lack of standards, this adds interoperability issues.

$5 \quad$ Lack of testing specifications.

Network solutions to offer Cordless Mobility are not yet consolidated, this implies the unavailability of test suites for network service features which have to be developed from scratch, using the service description. Regarding the DECT access, it must be remarked that the existing standardized (e.g. pr-TBR22 [3]) test suites are preliminary and need field verification. $6 \quad$ Lack of adequate testing tools.

For DECT testing, a number of tools exist for physical level (radio) verification. However they are only partially adequate for network integration testing, which need upper layers monitoring and simulations, as well as terminal emulators and load traffic generators for RFP, HDB and $\mathrm{LE}$ in a laboratory environment.

\section{TEST ACTIVITIES}

To cope with the requirements of Cordless Mobility service testing, a test plan was agreed between Italtel, Telecom Italia and CSELT, in order to share the effort of network integration activities.

The consolidated expertise gained by the partners in IN and switch testing (see [5], [6] and [7]) was useful to formulate an adequate plan, whose objective was to prove the appropriate behaviour of the systems in a controlled environment, before eventual deployment of the nodes in the real network for field experiments.

The testing activities were performed, for a three month period, in test plants located in Italtel, Telecom and CSELT laboratories.

The major items covered in the plan included:

- DECT GAP protocol conformance testing;

- HDB CCS7 and OA\&M testing;

- RFP functional testing; 
- LE OA\&M testing;

- end to end Cordless Mobility service testing;

- HDB verification under traffic conditions;

- LE verification under traffic condition.

Different test configurations were used, depending on the specific testing activity. End to end service verification required, as expected, the most complex testing configuration, shown in the following Figure 2.

For the in-lab testing activities, SSP functions were enabled on a separate UT Exchange (referred to as UT LE in the Figure 2), while CCFP and VDB capabilities on another node (UT DECT in the Figure 2).

Moreover, HDB capabilities were distributed in two physical entities: user profiles were managed by a mirrored national IN SCP node, while mobility management and authentication capabilities were managed on a dedicated "GSM like" HLR/AuC node upgraded with DECT authentication algorithms and $\mathrm{CM}$ user management. Without substantial changes to service profile requirements, this configuration added complexity to the tested network.

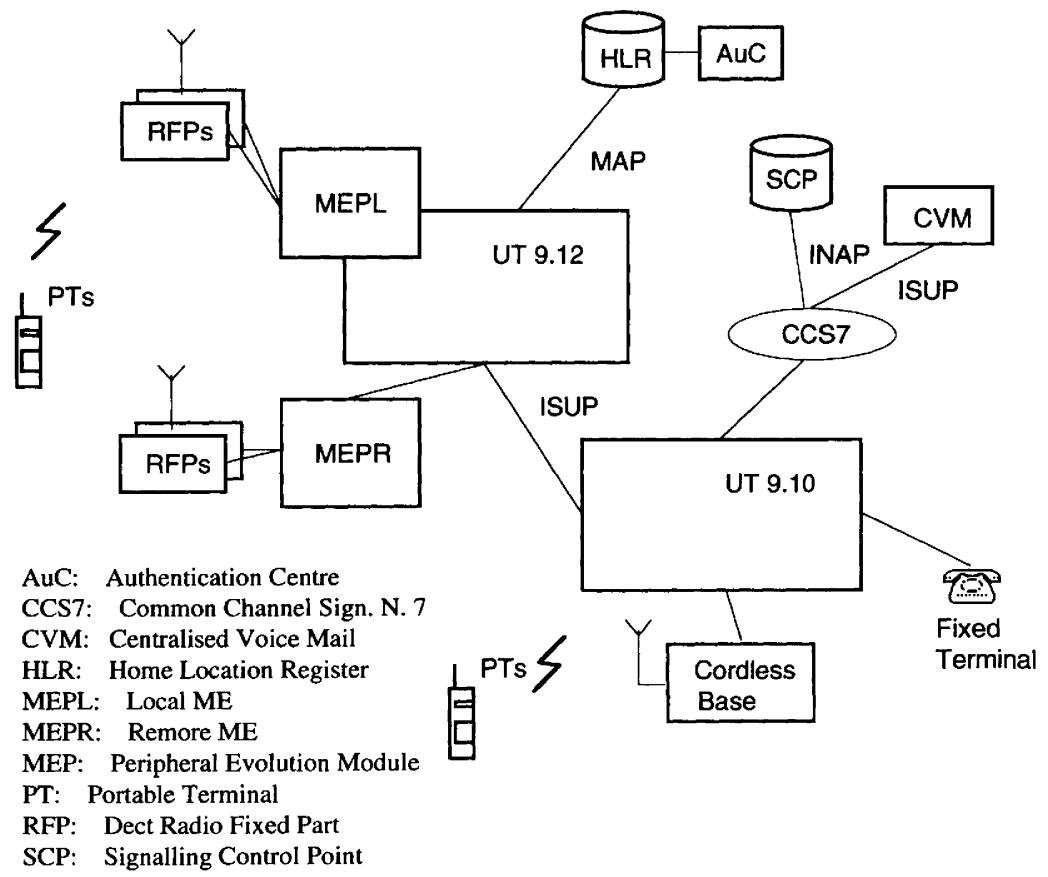

Figure 2 Test configuration for CM service integration testing. 


\subsection{DECT Protocol Testing}

This activity, finalized to verify the conformance of DECT implementation on the network elements, was based on ETSI standard pr-TBR22 [3]. The objective of the TBR (Technical Basis for Regulation) is to ensure interoperability of DECT GAP implementations at the air interface and comprises type approval tests for both FP (Fixed Part) and PP (Portable Part). In particular, only the FP test cases with reference to Network Layer (NWKL) were used in the service validation test session, as the data link layer (DLC) and the layers below (Medium Access Control Layer MAC and Physical Layer PHL) were object of detailed system integration testing performed during the LE and RFP design.

\section{Abstract Test Suite}

The NWKL pr-TBR22 test suite for FP is an ISO 9646 conformant ATS (Abstract Test Suite) written in TTCN notation and is composed by 84 test cases. In accordance with the DECT features supplied by UT100 a test case selection was made. In particular the following NWKL features were considered:

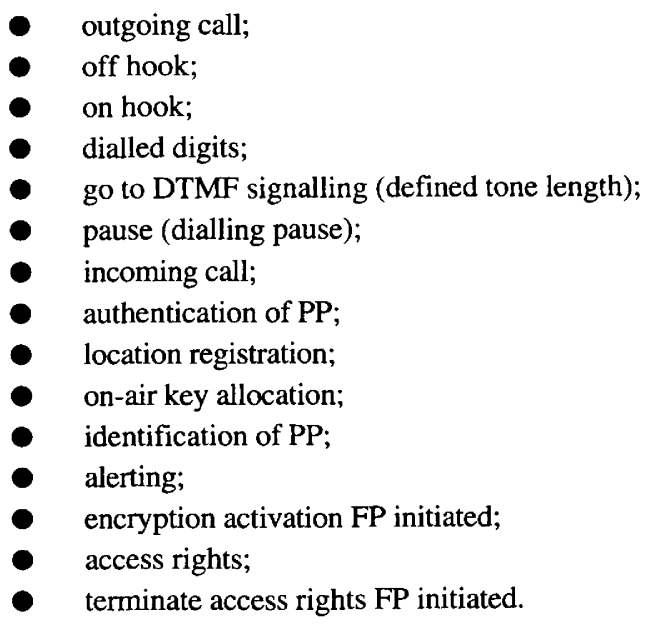

About 50 test cases were selected and executed. The excluded 34 test cases were related to features not supported on UT100 LE (i.e. On-Air Key Allocation and Access Rights procedures). Extra test cases needed to be added for a better coverage of the IUT capabilities in terms of error handling (i.e. test cases conceived for UT100 behaviour verification with DECT NWKL layer messages containing wrong information elements) and DECT NWKL layer procedure coexistence, as required in [4].

\section{Execution Environment}

The basic test equipment chosen for DECT GAP protocol conformance testing was a commercially available modular platform for the development and realization of DECT Executable Test Suites (ETS). The tool, through a socket connection mechanism on UNIX environment, communicates with a DECT front-end, and is essentially composed of a test run manager that can be activated via a graphic user interface based on Motif. Moreover the testing system includes all the ETSI pr-TBR22 test cases executable form for DECT Network layer.

The platform supplies the executable form of ETSI pi-TBR22 ATS for DECT in an enhanced 
' $C$ ' language. In particular, a macro has been defined for each TTCN primitive, in such a way that translation from TTCN to source code is simplified. After compilation, tests can be run directly on the tester and both trace and verdict are stored in a file.

The tests on UT100 LE were performed using a standalone network configuration as shown in the Figure 3.

In this network configuration, to allow optimal use of laboratory resources, instead of a real intelligent node, an HDB Emulator, developed by CSELT, supplied mobility management functions. The emulator reproduces the HDB behavicur at the HDB-VDB interface for a large set of scenarios, and can handle simultaneous transactions originated by up to 4 VDBs.

Moreover, a spectrum analyzer was used to monitor radio bearers during a DECT communication.

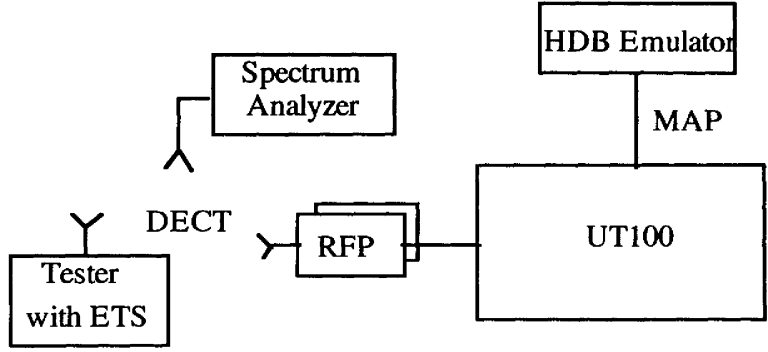

Figure 3 UT100 LE DECT protocol testing: network configuration.

The test session was useful to prove both the ATS and the ETS, at their first field usage. In particular some flaws were discovered on the ATS, promptly forwarded to ETSI through the test tool supplier. Moreover, as ATS coverage was considered not optimal, especially for protocol error handling, coexistence of $\mathrm{CC}$ (Call Control) and MM (Mobility Management) procedures, MM procedures nesting, additional test cases were added to the initial plan.

\section{Solution for data link layer (DLC) testing}

As DLC is deeply involved in connection handover procedure, a special testing configuration was set-up by the Italtel system integration department. This configuration, in order to assure a reliable and deterministic reproduction of handover and verify switch behaviour, bypasses the air interface by means of a RFP emulator directly connected to the RFP/LE interface. The DLC features under observation in this configuration were:

- basic data link capabilities;

- conformance to the relevant protocol standard (checksum calculation, fragmentation, retrasmission etc.);

- coordination with other functionalities in order to perform connection handover;

- evaluation and tuning of response time.

Only basic DLC capability testing was completed before the network testing took place, while the other specific features (i.e. DLC error handling), with a less impact to network wide 
functional verifications of all involved systems, were analyzed in parallel with end to end service test session.

\subsection{HDB Testing}

CM users' profiles are contained in the HDB, including also mobility management functionalities (Location Registration and Authentication). Careful attention was paid to the new capabilities required for mobility management with DECT access, while SCP functionalities were already verified in previous testing sessions for IN deployment. The HDB testing program covered CCS7 protocols, OA\&M procedures and HDB evaluation under traffic conditions.

\section{HDB CCS7 Protocol Testing}

HDB dialogues with Visitor Data Bases (VDB) through a CCS7 protocol stack: MTP (Message Transfer Part), SCCP (Signalling Connection Control Part), TCAP (Transaction Capabilities Application Part), MAP (Mobile Application Part).

For MTP testing a Test Suite developed by CSELT in a CEC sponsored CTS project was adopted. The Abstract Test Suite (ATS), based on ITU Q.781 and Q.782 and recommendations, written in TTCN notation, includes over 300 tests, 170 of which applicable to the IUT; the Executable Test Suite was obtained using a tool which translates the TTCN MP code into the test source code of a commercial protocol tester.

SCCP and TCAP testing were based on two existing Test Suites, both entirely developed by CSELT using TTCN notation, already used for IN qualification activities. The SCCP Test Suite covers connectionless service functions as well as management aspects, and includes over 100 tests. As for TCAP Test Suite, it includes about 170 test cases.

As for Application layer protocol testing, with respect to the general objective to guarantee interoperability, it was considered sufficiently covered by end to end service testing and consequently no additional tests were specified and executed.

\section{$O A \& M$}

The objective was to verify the procedures related to system administration and CM service management; in particular the following aspects were considered:

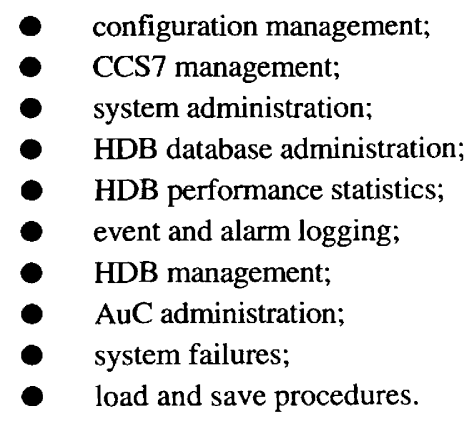

About 180 tests cases were specified in textual notation and performed in a stand-alone configuration using the user interface provided by the system under test. For some tests requiring background traffic (HDB performance statistics) a VDB traffic emulator (similar to the already cited HDB emulator) developed by CSELT on a commercial protocol tester was used. 


\section{$H D B$ evaluation under traffic condition}

HDB evaluation under traffic conditions focused on capacity evaluation for different traffic types, response delay at different traffic levels, impact of administrative activities on call processing and system behaviour under degraded system configurations.

Tests were executed to verify system capacity and CCS7 response time with respect to four different call scenarios: location registration, authentication parameters request, incoming call handling and a combination of all of them (traffic mix). In the lack of other data, two different traffic models were considered, one based on GSM recommendations, and the other based on provisional Cordless Mobility traffic forecasts: they mainly differed for Location registration activity and user's call frequency.

Regarding administrative tests, a number of operator's actions were examined under different traffic conditions, to evaluate the system administration time. As CM is conceived for a large residential market, administration activities as introduction/removal of users from the database, trace activation/deactivation, $\log$ files activation/deactivation could be demanded.

Robustness and stability tests allowed to determine the call loss rate and the HLR behaviour in overload conditions and during restoration procedures.

Figure 4 shows the test configuration. A MAP traffic generator and a protocol tester emulated each one a couple of VDBs. The generator was based on a software package commercially available for GSM testing, while the protocol tester, used for sample traffic on which to perform response delay measurements, was based on the cited VDB emulation package developed by CSELT.

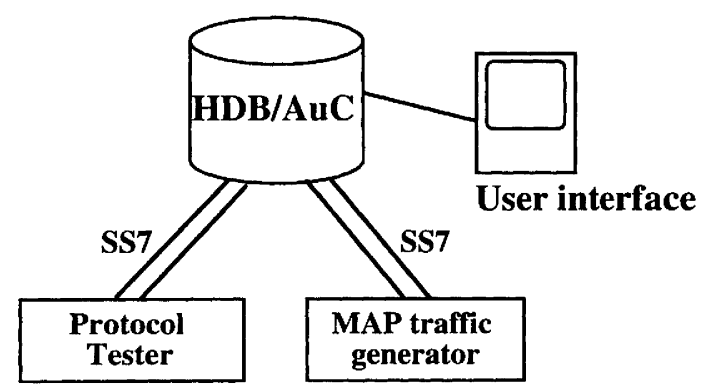

Figure 4 HDB performance test configuration.

\subsection{RFP Protocol and Functional Testing}

In the experimented architecture, the RFP manages the MAC protocol and controls radio communication with the portable part. In the upstream direction the RFP communicates with the local exchange through an ISDN like interface using a proprietary adaptation layer able to convey MAC abstract primitives on the physical interface. Through the same interface also all the information related to the RFP OA\&M procedures are transported.

Due to the complexity of the RFP functionalities, within Italtel and CSELT, an ad-hoc group responsible for the validation of the RFP itself was created. In order to be able to distinguish any problems due to the LE from any problems due to the RFP, a completely stand alone test 
configuration was setup, with the development of a local exchange emulator and a portable part emulator. This approach was specially iiis !!sed to reproduce, in a controlled environment, the following procedures:

- basic communication capabilities of the ISDN-like interface;

- downloading of the RFP software and ce infiguration;

- RFP operational state control;

- radio and MAC layer capabilities.

The stand alone configuration for RFP testing was really useful in understanding and solving in a very short time the problems arisen during end to end service validation, allowing for parallel activities.

\subsection{Local Exchange OA\&M Testing}

$\mathrm{CM}$ service required the development of a wide set of maintenance and operational procedures on the UT 100 local exchange. Some of these new functionalities were tuned in a strict cooperation between RFP and local exchange testing engineers. About 300 test cases were specified and detailed, in a textual notation, on the basis of supplier's documentation, covering various OA\&M aspects. In particular, the functions related with RFP software downloading, RFP configuration and RFP operational states management made use of a special testing configuration which adopted the powerful self monitoring capabilities active on both local exchange UT DECT and RFP.

The verification of the management of the new DECT related functionalities inserted in the existing UT100 architecture as charging record generation, traffic measurement control, system and network configuration was performed in the traditional step by step way, with special care in the measurement of the new synchronization capabilities necessary for handover scenarios.

\subsection{End to End Service Integration Testing}

\section{Objectives and Methodology}

Although essential to anticipate problems, standalone test activities does not always reveal all interoperability problems in the network. In fact, with the presence of many network elements (in this case PT and RFP DECT, UT100 LE, network databases HDB and SCP), manufactured by different suppliers, subtle variations in interpreting and implementing service specifications can originate interoperability problems. In addition, use of DECT access technique for public applications is relatively new and, in many cases (i.e. signalling procedures for handover), technical specifications are too loose and imprecise.

To verify system interoperability, an extensive activity was devoted by CSELT to end-to-end CM service integration testing. These tests are conceived as network wide functional verifications of all involved systems before their field application with real users. They are focused on the verification of interoperability between CM nodes, the complex interaction between the different network protocols (interworking between MAP, INAP, ISUP and DECT), and the analysis of DECT impact on the switch and user's service perception.

To achieve the objective, the following methodology has been adopted:

1. thorough analysis of service specifications to identify all relevant user's actions and behaviours, including unexpected events;

2. detailed analysis of all relevant network field configurations: RFP configurations, exchange set-up, location and handover domains; 
3. cross analysis between the set of service scenarios and network field configurations, to derive the relevant test cases and choose the appropriate test plant configuration;

4. identification of a test suite structure, test purposes and test details;

5. identification of test suite execution methods at the test plant.

The early definition of the test suite and its discussion within the testing team, was proven very useful to integrate manufacturer's documentation for the LE, the RFP, the HDB, the DECT GAP terminal, highlighting ambiguities and anticipating interoperability problems.

\section{Test Suite and Test Purposes (TP) specification}

Based on the previously defined methodology, a Test Suite has been defined using traditional test description. Use of TTCN or other more formal methods was not considered as service specifications were not described in formai way and due to time constraints.

Each TP was specified using a test management tool developed by CSELT and called MITS (Multi Integrated Test System). Test specification layout includes a short test purpose description, some information about network nodes under test, execution environment configuration, test details and the expected final status. Test details include a detailed view of the expected information flow (application operations, messages and parameters) and events at the examined network and user interfaces.

The developed test suite was structured into two main test groups (Service Handling and User Procedures), and comprised abou! 250 test cases. Each test group was structured in subgroups covering the items indicated in Table 2:

Table2 Test group included in the Test Suite

\begin{tabular}{|c|c|}
\hline Service Handling & $\begin{array}{l}\text { Mobility Management } \\
\text { Call Handling } \\
\text { Restoration } \\
\text { Handover } \\
\text { Centralized Voice Mail }\end{array}$ \\
\hline User Procedures & $\begin{array}{l}\text { Public/Private switch proc. } \\
\text { Cordless usage }\end{array}$ \\
\hline
\end{tabular}

- Mobility Management, Call Handling and Handover: tests for mobility management, call control and handover verification procedures.

- Restoration: tests for network nodes (databases) out-of-service restoration procedures.

- Centralized Voice Mail: tests for verification of the correct behaviour of the centralized voice mail, when involved.

- User Procedures: tests for handset use verification in private environment and change of network access method proofs (form public to private and vice-versa) performed by the $\mathrm{CM}$ user.

The tests were mainly executed with manual procedures, using standard terminals and then comparing the results of monitoring tools at the various interfaces. For some tests, a prototypal DECT GAP terminal emulator provided by Necsy was useful to reproduce user's actions and force events. The emulator supports the following functions:

- monitoring of the radio interface with one or more RFP (Radio Fixed Part), with complete decoding capabilities;

- manual forcing of attach, handover and anomalous situations; 
- file storage of collected data;

- displaying of radio-electric parameters and RFP info channels.

This test session was probably one of the most fruitful ones, as it was essential not only to verify the interoperability of all systems, but also how service could be perceived by customers, providing a number of useful suggestions to improve user procedures, timing, and messages on terminal display.

\subsection{UT100 evaluation under traffic condition}

Load testing activity aimed at obtaining the following main goals:

- to give a preliminary system performance evaluation of Italtel UT100 local exchange, focusing the attention on the specific subsystems which support wireless access capabilities, DECT call control, mobility management procedures and local data base;

- to observe behaviour and stability of the network, by simulating traffic conditions in the laboratory environment.

Tests reproduced traffic scenarios involving both mobile terminals and POTS subscribers, and various services offered to the subscriber, like the CVM in case of called subscriber not reachable, or the possibility to switch to the home-co-dless environment.

The test plant network configuration was identical to that used for end to end service integration testing and shown in Figure 2.

The activity required the utilization, in the laboratory environment, of both portable terminals (GAP profile) and a new DECT automatic traffic generator called DTLS (C) (DTLS is a trademark of Necsy). The generator can perform location registration procedures and simulate originating/terminating call scenarios from/to a relevant number of DECT terminals. DTLS has a modular architecture, with a variable number of radio modules which simulate mobile terminals, and a variable number of modules emulating POTS subscriber lines. A control unit allows to program a testing session with the desired traffic mix, collects data and produces session reports. Useful data are available, like call loss rate, failure causes, call tracing, and so on. The session was also useful to improve the prototypal version of the tool and identify new requirements for more extensive test campaigns.

The activity allowed to identify problems related to traffic conditions, which affected the quality of service offered to the CM subscriber and helped in their resolution. Nevertheless, the complexity of service architecture, and the fact that neither terminals, nor traffic tools are completely certified gave rise to troubles in correctly finding out which subsystem causes malfunctions to happen, in order to help a timely deployment of solutions.

\section{CONCLUSIONS}

This paper has described the testing approach chosen for the validation of a CM solution, based on DECT radio access technique, integrated into the Italtel UT100 local exchanges. The objective was not only to gain the confidence that the service is technically robust and acceptable to end users, but also the assurance that CM service would not adversely affect normal PSTN network operations. A side objective was also to verify the adequacy of testing tools and procedures for CM service.

The CM testing approach must cope with new challenges with respect to "traditional" switch based service testing needs, involving various technical areas as: integration of wireless and wireline access to switching system, interaction between different and complex mobile and network protocols in the network, use of newly released portable handsets and introduction of new network systems. This requires a combination of testing strategies already in use for IN 
and GSM testing, but with an extra level of complexity in network integration.

To cope with CM service testing requirements, a complex test plant configuration was designed and set-up, combining both radio (DECT) and network (PSTN, IN, GSM) skills in an unique team and paying special care in highlighting interoperability issues. Different test configurations were then used, sometimes in parallel testing sessions. Some simplifications and a significant saving of time were possible by a strict cooperation with the Italtel system integration department, which avoided unnecessary test duplications. This interdisciplinary approach and the establishment of such a complete test environment were proven essential to achieve the testing objectives in the scheduled time.

Protocol testing was performed extensively only on a subset of the network elements, leaving to the end-to-end service validation tests the important task of verifying the entire CM network as a whole. In the lack of standards for CM service, a new test suite needed to be developed, while for protocol aspects preliminary TTCN ATS were used.

During the activity evolution, some critical aspects had to be tackled, as the lack of adequate coverage in the available ATS for DECT GAP testing specifications. As for testing tools, several of them had to be prepared with in-lab developments. In general, there is still a lack of adequate testing tools for DECT radio interface as traditional DECT tools are only partially adequate for network integration testing and more appropriate for radio measurements. This means that identifying, tracing and debugging problems related to DECT upper layers had to rely mostly on the experience and intuition of the operators. Some prototypal DECT radio interface monitor and simulator instruments were then procured and have been extremely improved as a result of this activity. Nevertheless, a number of key issues have to be further studied for more intensive testing activities as those which could be required by a large deployment of the systems in the network, in particular for DECT traffic generation at very high traffic rates (e.g. thousands of Busy Hour Calls), and automatic execution of end to end service tests.

\section{ACKNOWLEDGEMENTS}

The authors wish to thank the colleagues who have contributed to the testing activities and the revision of the paper, in particular Silvio Grande and Stefano Gollinucci, from CSELT, and Renzo dal Molin and Giuseppe della Valle from Italtel.

\section{REFERENCES}

[1] Eynard et alii "Local Mobility and Personal communication services: market aspects and network solutions", ISS 95.

[2] Faraci, Pietroiusti, Santagostino "IN based approach for the provision of PCS services using PSTN infrastructure", ICIN'94.

[3] ETSI RES3 T, prTBR 22.

[4] ETSI prETS 300444 "Radio Equipment and Systems (RES); Digital European Cordless Telecommunications (DECT) Generic Access Prufilc (GAP)", ETSI, Sophia Antipolis, Valbonne, F, February 1995.

[5] E. Guarene, P. Marchese "Advanced testing tools for the Italian IN", IWPTS 1991, Leidschendam, NL.

[6] G.P. Fici, P. Marchese, S. Orlando "SCP performance testing and evaluation", ICIN'94, Bordeaux, F, October 1994.

[7] L. Dragoni, L. Gabrielli, P. Marchese "Intelligent Network Service Testing", ICCC Conference on IN "The path to global Networking", Tampa, USA, May 1992.

[8] R0912-REP-DECT (Italtel confidential documentation), Italtel, Castelletto di Settimo Milanese (MI), I.

[9] ETSI Recommendation GSM 09.02 v. 3.8.0 "Mobile Application Part Specification", ETSI, Sophia Antipolis, Valbonne, F, January 1991. 


\section{ABBREVIATIONS}

ADPCM: Adaptive Differential Pulse Code Modulation

AuC: $\quad$ Authentication Centre

ATS: $\quad$ Abstract Test Suite

BHC: $\quad$ Busy Hour Calls

BRA: Base Rate Access

CC: Call Control

CCFP: $\quad$ Common Control Fixed Part

CCS7: $\quad$ Common Channel Signalling number 7

CM: $\quad$ Cordless Mobility

CS-1: $\quad$ Capability Set 1

CSELT: Centro Studi e Laboratori Telecomunicazioni s.p.a.

CTM: $\quad$ Cordless Terminal Mobility

CVM: $\quad$ Centralized Voice Mail

DECT: Digital European Cordless Telecommunications

DLC: $\quad$ Data Link Control layer

DTMF: Dual Tone Multiple Frequency

ETS: $\quad$ Executable Test Suite

ETSI: $\quad$ European Telecommunication Standard Institute

FP: $\quad$ Fixed Part

GAP: $\quad$ Generic Access Profile

GSM: Global System for Mobile teleconumunications

HDB: $\quad$ Home Data Base

HLR: $\quad$ Home Location Register

IN: Intelligent Network

INAP: Intelligent Network Application Part

ISDN: Integrated Services Digital Network

ISO: International Standard Organisation

ISUP: $\quad$ ISDN User Part

IUT: Interface Under Test

LE: Local Exchange

MAC: $\quad$ Media Access Control

MAP: $\quad$ Mobile Application Part

MITS: $\quad$ Multi Integrated Test System on personas computer

MM: $\quad$ Mobility Management

MSRN: $\quad$ Mobile Station Roaming Number

MTP: $\quad$ Message Transfer Part

NWKL: $\quad$ NetWorK Layer

OA\&M: $\quad$ OperAtion \& Maintenance

PHL: $\quad$ PHisical Layer

POTS: $\quad$ Plain Ordinary Telephone Services

PP: $\quad$ Portable Part

PSTN: $\quad$ Public Switched Telephone Network

RFP: $\quad$ Radio Fixed Part

SCCP: $\quad$ Signalling Connection Control Part

SCP: $\quad$ Service Control Point

SSP: $\quad$ Service Switching Point

TBR: Technical Basis for Regulation

TCAP: $\quad$ Transaction Capability Application Part

TP: $\quad$ Test Purpose

TS: $\quad$ Test Suite

TTCN: $\quad$ Tree and Tabular Combined Notation

VDB: $\quad$ Visitor Data Base 